\title{
The (G'/G)-Expansion Method for the Sine-Gordon Equation, Sinh-Gordon Equation and Liouville Equaiton
}

\author{
Hui-Ju DAI and Lian-Zhong LI* \\ School of Science, Jiangnan University \\ Wuxi, Jiangsu 214122, PR China \\ 923649959@qq.com; Ilz3497@163.com
}

\begin{abstract}
Keywords: (G'/G)-expansion method, Traveling wave solution, Sine-Gordon equation,
\end{abstract} Sinh-Gordon equation, Liouville equation.

\begin{abstract}
In this paper, the $\left(\mathrm{G}^{\prime} / \mathrm{G}\right)$-expansion method is applied to find the exact solutions for Sine-Gordon equation, Sinh-Gordon equation and Liouville equaiton. The (G'/G)-expansion method is an effective method in investigating exact traveling wave solutions to nonlinear evolution equations (NLEEs) in the field of applied mathematics, mathematical physics and engineering.
\end{abstract}

\section{Introduction}

The nonlinear evolution equation $u_{x t}-h(u)=0 \quad$ (1.1) has important applications in many scientific fields such as nonlinear optics, solid state physics and quantum field theory, where $u=u(x, t)$ is the unknown function of the space variable $x$ and time $t$, and the function $h(u)$ may be

$$
h(u)=\sin (m u), \sinh (m u), e^{m u}, \cdots
$$

which correspond respectively to the sine-Gordon equation[1]

$$
u_{x t}=\sin (m u),
$$

Sinh-Gordon equation[2]

$$
u_{x t}=\sinh (m u),
$$

Liouville equation[3]

$$
u_{x t}=e^{m u} .
$$

The Sine-Gordon equation is Lax integrable and has nontrivial prolongation structures, which plays an important role in many scientific fields and nonlinear optics. The sinh-Gordon equation is also a Lax integrable system and possesses similarity reductions to third Painlevé equation. It is worth noting that by using the identity $\mathrm{e}^{\mathrm{mu}}=\sinh (m u)+\cosh (m u)$, Liouville equation becomes a combined sinh-cosh-Gordon equation[4]

$$
u_{x t}=\sinh (m u)+\cosh (m u) .
$$

These equations have been investigated by different methods, and some exact solutions are derived [5-8]. However, there are still many other exact solutions to be found. In this paper, we will derive more new exact solutions based on $\left(\mathrm{G}^{\prime} / \mathrm{G}\right)$-expansion method.

\section{Description of the (G'/G)-expansion Method}

First, we simply describe the (G'/G)-expansion method. For the equation

$$
P\left(u, u_{x}, u_{t}, u_{x x}, u_{x t}, u_{t t}, \cdots\right)=0 \text {, }
$$

where $P$ is a polynomial in its arguments. The transformation $u(\mathrm{x}, \mathrm{t})=f(\xi), \xi=k x-c t$, reduces Eq.(2.1) to the ordinary differential equation

$$
P\left(f, k f^{\prime}, c f^{\prime}, k^{2} f^{\prime \prime}, k c f^{\prime \prime}, c^{2} f^{\prime \prime}, \cdots\right)=0,
$$


where $f=f(\xi)$ and the prime denotes derivative with respect to $\xi$. We assume that the solution of Eq.(2.2) can be expressed by a polynomial in $\left(\mathrm{G}^{\prime} / \mathrm{G}\right)$ as follows:

$$
f=\sum_{i=1}^{n} a_{i}\left(\frac{G^{\prime}}{G}\right)^{i}+a_{0}
$$

where $G=G(\xi)$ is the solution of the auxiliary linear second-order ordinary differential equation

$$
G^{\prime \prime}+\lambda G^{\prime}+\mu G=0
$$

where $G^{\prime}=\frac{d G}{d \xi}, G^{\prime \prime}=\frac{d^{2} G}{d \xi^{2}}, \quad a_{n} \neq 0, \cdots, a_{1}, a_{0}, \lambda$ and $\mu$ are constants to be determined later. Using the general solutions of Eq.(2.4), we have

$$
\frac{G^{\prime}(\xi)}{G(\xi)}=\left\{\begin{array}{l}
\frac{\sqrt{\lambda^{2}-4 \mu}}{2}\left(\frac{C_{1} \sinh \left(\frac{1}{2} \sqrt{\lambda^{2}-4 \mu \xi}\right)+C_{2} \cosh \left(\frac{1}{2} \sqrt{\lambda^{2}-4 \mu \xi}\right)}{C_{1} \cosh \left(\frac{1}{2} \sqrt{\lambda^{2}-4 \mu \xi}\right)+C_{2} \sinh \left(\frac{1}{2} \sqrt{\lambda^{2}-4 \mu \xi}\right)}\right)-\frac{\lambda}{2}, \lambda^{2}-4 \mu>0, \\
\frac{\sqrt{4 \mu-\lambda^{2}}}{2}\left(\frac{-C_{1} \sin \left(\frac{1}{2} \sqrt{4 \mu-\lambda^{2} \xi}+C_{2} \cos \left(\frac{1}{2} \sqrt{4 \mu-\lambda^{2} \xi}\right.\right.}{C_{1} \cos \left(\frac{1}{2} \sqrt{4 \mu-\lambda^{2} \xi}+C_{2} \sin \left(\frac{1}{2} \sqrt{4 \mu-\lambda^{2} \xi}\right.\right.}\right)-\frac{\lambda}{2}, \lambda^{2}-4 \mu<0,
\end{array} \quad \mathrm{~V}\right.
$$

where $\mathrm{C}_{1}, \mathrm{C}_{2}$ are arbitrary constants.

\section{Exact Traveling Wave Solution of the Equations}

Now, we will find the traveling wave solutions of the equations for Eq.(1.3), we employ the transformation:

$$
y(x, t)=e^{i m u},
$$

for Eq.(1.4) and Eq.(1.5) we employ the transformation:

$$
y(x, t)=e^{m u},
$$

where $i^{2}=-1$ from Eq.(3.1), we get

$$
u=\frac{1}{m} \arccos h \frac{y^{2}+1}{2 y}
$$

and

$$
u=\frac{1}{m} \arccos h \frac{y^{2}+y^{-2}}{2} .
$$

Using the conversion formula, we transform (1.3)and (1.4) into the following equation

$$
2 y y_{x t}-2 y_{x} y_{t}-m y^{3}+m y=0,
$$

and Eq.(1.5) is equivalent to the following equation

$$
y y_{x t}-y_{x} y_{t}-m y^{3}=0 .
$$

Now, we will find the traveling wave solutions of the Eq.(3.3) and Eq.(3.4) instead of Eq.(1.3), Eq.(1.4) and Eq.(1.5) by using the $\left(\mathrm{G}^{\prime} / \mathrm{G}\right)$-expansion method.

\section{Application of the Method to the Eq. (3.3)}

In order to obtain the exact traveling wave solutions of the $E q(3.3)$, let

$$
y(x, t)=f(\xi), \xi=k x-c t,
$$

then Eq.(3.3) become:

$$
-2 c k f f^{\prime \prime}+2 c k\left(f^{\prime}\right)^{2}-m f^{3}+m f=0 .
$$

in order to balance the term $f^{3}$ and $f f^{\prime \prime}$ in Eq.(3.6), we get $n=2$. Therefore, the solution of $\mathrm{Eq}(3.6)$ takes the form: 


$$
f=a_{2}\left(\frac{G^{\prime}}{G}\right)^{2}+a_{1}\left(\frac{G^{\prime}}{G}\right)+a_{0}, a_{2} \neq 0
$$

Substituting (3.7) into (3.6) and setting the coefficients of $\left(\frac{G^{\prime}}{G}\right)^{i}, 0 \leq i \leq 6$, to zero, we derive a set of algebraic equations for $a_{0}, a_{1}, a_{2}, c, k$ and $m$ :

$$
\begin{gathered}
2 c k\left(a_{1}^{2} \mu^{2}-a_{0} a_{1} \lambda \mu-2 a_{0} a_{2} \mu^{2}\right)-m a_{0}^{3}+m a_{0}=0, \\
2 c k\left(a_{1}^{2} \lambda \mu+2 a_{1} a_{2} \mu^{2}-a_{0} a_{1} \lambda^{2}-2 a_{0} a_{1} \mu-6 a_{0} a_{2} \lambda \mu\right)-3 m a_{0}{ }^{2} a_{1}+m a_{1}=0, \\
2 c k\left(a_{1} a_{2} \lambda \mu+2 a_{2}^{2} \mu^{2}-4 a_{0} a_{2} \lambda^{2}-3 a_{0} a_{1} \lambda-8 a_{0} a_{2} \mu\right)-3 m a_{0}{ }^{2} a_{2}-3 m a_{0} a_{1}^{2}+m a_{2}, \\
2 c k\left(-2 a_{1} a_{2} \mu-a_{1}{ }^{2} \lambda-a_{1} a_{2} \lambda^{2}+2 a_{2}{ }^{2} \mu \lambda-2 a_{0} a_{1}-10 a_{0} a_{2} \lambda\right)-6 m a_{0} a_{1} a_{2}-m a_{1}^{3}=0, \\
2 c k\left(-5 a_{1} a_{2} \lambda-a_{1}^{2}-6 a_{0} a_{2}\right)-3 m a_{0} a_{2}^{2}-3 m a_{1}^{2} a_{2}=0, \\
2 c k\left(-4 a_{1} a_{2}-2 a_{2}{ }^{2} \lambda\right)-3 m a_{1} a_{2}^{2}=0, \\
-4 c k a_{2}{ }^{2}-m a_{2}{ }^{3}=0 .
\end{gathered}
$$

Solving the set of algebraic equations by use of Maple, we get the following results:

$$
\begin{gathered}
\text { case } 1: a_{0}=\frac{-\lambda^{2}}{\lambda^{2}-4 \mu}, a_{1}=\frac{-4 \lambda}{\lambda^{2}-4 \mu}, a_{2}=\frac{-4}{\lambda^{2}-4 \mu}, c=\frac{m}{k \lambda^{2}-4 k \mu} . \\
\text { case } 2: a_{0}=\frac{\lambda^{2}}{\lambda^{2}-4 \mu}, a_{1}=\frac{4 \lambda}{\lambda^{2}-4 \mu}, a_{2}=\frac{4}{\lambda^{2}-4 \mu}, c=-\frac{m}{k \lambda^{2}-4 k \mu} .
\end{gathered}
$$

\section{Case 1}

When $\lambda^{2}-4 \mu>0$, we obtain hyperbolic function solution:

$$
f_{11}=-\left(\frac{C_{1} \sinh \left(\frac{1}{2} \sqrt{\lambda^{2}-4 \mu \xi}\right)+C_{2} \cosh \left(\frac{1}{2} \sqrt{\lambda^{2}-4 \mu \xi}\right)}{C_{1} \cosh \left(\frac{1}{2} \sqrt{\lambda^{2}-4 \mu \xi}\right)+C_{2} \sinh \left(\frac{1}{2} \sqrt{\lambda^{2}-4 \mu \xi}\right)}\right)^{2} .
$$

When $\lambda^{2}-4 \mu<0$, the trigonometric function solution of Eq. (3.3) is:

$$
f_{12}=\left(\frac{-C_{1} \sin \left(\frac{1}{2} \sqrt{4 \mu-\lambda^{2} \xi}\right)+C_{2} \cos \left(\frac{1}{2} \sqrt{4 \mu-\lambda^{2} \xi}\right)}{C_{1} \cos \left(\frac{1}{2} \sqrt{4 \mu-\lambda^{2} \xi}\right)+C_{2} \sin \left(\frac{1}{2} \sqrt{4 \mu-\lambda^{2} \xi}\right)}\right)^{2},
$$

where $\xi=k x-c t, \quad c=\frac{m}{k \lambda^{2}-4 k \mu}, \quad C_{1}, \quad C_{2}$ and $k$ are arbitrary constants.

In view of Eqs.(3.2), we get the exact explicit traveling wave solution of the sine-Gordon equation are as follows:

$$
u_{111}=\frac{1}{m} \arccos h \frac{\left(f_{11}\right)^{2}+1}{-2 f_{11}}, \text { and } u_{112}=\frac{1}{m} \arccos h \frac{\left(f_{12}\right)^{2}+1}{2 f_{12}} .
$$

and the exact traveling wave solution of the sine-Gordon equation are as follows:

$$
u_{211}=\frac{1}{m} \arccos h \frac{\left(f_{11}\right)^{2}+\left(f_{11}\right)^{-2}}{2} \text { and } u_{211}=\frac{1}{m} \arccos h \frac{\left(f_{12}\right)^{2}+\left(f_{12}\right)^{-2}}{2} \text {. }
$$

\section{Case 2}

When $\lambda^{2}-4 \mu>0$, we obtain hyperbolic function solution:

$$
f_{21}=\left(\frac{C_{1} \sinh \left(\frac{1}{2} \sqrt{\lambda^{2}-4 \mu \xi}\right)+C_{2} \cosh \left(\frac{1}{2} \sqrt{\lambda^{2}-4 \mu \xi}\right)}{C_{1} \cosh \left(\frac{1}{2} \sqrt{\lambda^{2}-4 \mu \xi}\right)+C_{2} \sinh \left(\frac{1}{2} \sqrt{\lambda^{2}-4 \mu \xi}\right)}\right)^{2} .
$$

when $\lambda^{2}-4 \mu<0$, the trigonometric function solution of Eq.(3.3) is: 


$$
f_{22}=-\left(\frac{-C_{1} \sin \left(\frac{1}{2} \sqrt{4 \mu-\lambda^{2} \xi}\right)+C_{2} \cos \left(\frac{1}{2} \sqrt{4 \mu-\lambda^{2} \xi}\right)}{C_{1} \cos \left(\frac{1}{2} \sqrt{4 \mu-\lambda^{2} \xi}\right)+C_{2} \sin \left(\frac{1}{2} \sqrt{4 \mu-\lambda^{2} \xi}\right)}\right)^{2},
$$

where $\xi=k x-c t, \quad c=-\frac{m}{k \lambda^{2}-4 k \mu}, C_{1}, C_{2}$ and $k$ are arbitrary constants.

In view of Eqs.(3.2), we get the exact explicit traveling wave solution of the sine-Gordon equation are as follows:

$$
u_{121}=\frac{1}{m} \arccos h \frac{\left(f_{21}\right)^{2}+1}{2 f_{21}} \text { and } u_{122}=\frac{1}{m} \arccos h \frac{\left(f_{22}\right)^{2}+1}{-2 f_{22}} \text {. }
$$

\section{Application of the Method to the Eq. (3.4)}

In order to obtain the exact traveling wave solutions of the $E q(3.4)$, let

$$
y(x, t)=f(\xi), \xi=k x-c t,
$$

then Eq (3.4) become:

$$
-c k f f^{\prime \prime}+c k\left(f^{\prime}\right)^{2}-m f^{3}=0 .
$$

Similarly, we get $\mathrm{n}=2$, suppose Eq.(3.16) has the following formal solutions:

$$
f=a_{2}\left(\frac{G^{\prime}}{G}\right)^{2}+a_{1}\left(\frac{G^{\prime}}{G}\right)+a_{0}, a_{2} \neq 0 .
$$

Then we derive a set of algebraic equations for $a_{0}, a_{1}, a_{2}, c, k$ and $m$ :

$$
\begin{gathered}
c k\left(\mathrm{a}_{1}{ }^{2} \mu^{2}-\mathrm{a}_{0} \mathrm{a}_{1} \lambda \mu-2 \mathrm{a}_{0} \mathrm{a}_{2} \mu^{2}\right)-\mathrm{ma}_{0}{ }^{3}=0, \\
c k\left(a_{1}{ }^{2} \lambda \mu+2 a_{1} a_{2} \mu^{2}-a_{0} a_{1} \lambda^{2}-2 a_{0} a_{1} \mu-6 a_{0} a_{2} \lambda \mu\right)-3 m a_{0}{ }^{2} a_{1}=0, \\
c k\left(\mathrm{a}_{1} \mathrm{a}_{2} \lambda \mu+2 \mathrm{a}_{2}{ }^{2} \mu^{2}-4 \mathrm{a}_{0} \mathrm{a}_{2} \lambda^{2}-3 \mathrm{a}_{0} \mathrm{a}_{2} \lambda-8 \mathrm{a}_{0} \mathrm{a}_{2} \mu\right)-3 m \mathrm{a}_{0}{ }^{2} a_{2}-3 m a_{0} a_{1}{ }^{2}=0, \\
c k\left(-2 a_{1} a_{2} \mu-a_{1}{ }^{2} \lambda-a_{1} a_{2} \lambda^{2}+2 a_{2}{ }^{2} \mu \lambda-2 a_{0} a_{1}-10 a_{0} a_{2} \lambda\right)-6 m a_{0} a_{1} a_{2}=0, \\
c k\left(-5 a_{1} a_{2} \lambda-a_{1}{ }^{2}-6 a_{0} a_{2}\right)-3 m a_{0} a_{2}{ }^{2}-3 m a_{1}{ }^{2} a_{2}=0, \\
c k\left(-4 a_{1} a_{2}-2 a_{2}{ }^{2} \lambda\right)-3 m a_{1} a_{2}{ }^{2}=0, \\
-2 c k a_{2}{ }^{2}-m a_{2}{ }^{3}=0 .
\end{gathered}
$$

Solving the set of algebraic equations, we get

$$
a_{0}=\frac{-2 \mu}{m}, a_{1}=\frac{-2 \lambda}{m}, \quad a_{2}=\frac{-2}{m}, \quad c=\frac{1}{k} .
$$

When $\lambda^{2}-4 \mu>0$, we obtain hyperbolic function solution:

$$
f_{31}=-\frac{\lambda^{2}-4 \mu}{2 m}\left(\frac{C_{1} \sinh \left(\frac{1}{2} \sqrt{\lambda^{2}-4 \mu \xi}\right)+C_{2} \cosh \left(\frac{1}{2} \sqrt{\lambda^{2}-4 \mu \xi}\right)}{C_{1} \cosh \left(\frac{1}{2} \sqrt{\lambda^{2}-4 \mu \xi}\right)+C_{2} \sinh \left(\frac{1}{2} \sqrt{\lambda^{2}-4 \mu \xi}\right)}\right)^{2}+\frac{\lambda^{2}-4 \mu}{2 m} .
$$

When $\lambda^{2}-4 \mu<0$, the trigonometric function solutions of $E q(3.4)$ can be obtained as follow:

$$
f_{32}=\frac{\lambda^{2}-4 \mu}{2 m}\left(\frac{-C_{1} \sin \left(\frac{1}{2} \sqrt{4 \mu-\lambda^{2} \xi}\right)+C_{2} \cos \left(\frac{1}{2} \sqrt{4 \mu-\lambda^{2} \xi}\right)}{C_{1} \cos \left(\frac{1}{2} \sqrt{4 \mu-\lambda^{2} \xi}\right)+C_{2} \sin \left(\frac{1}{2} \sqrt{4 \mu-\lambda^{2} \xi}\right)}\right)^{2}+\frac{\lambda^{2}-4 \mu}{2 m},
$$

where $\xi=k x-c t, \quad c=\frac{m}{k \lambda^{2}-4 k \mu}, \quad C_{1}, C_{2}$ and $k$ are arbitrary constants.

Then we get the exact traveling wave solution of the Liouville equation:

$$
u_{331}=\frac{1}{m} \arccos h \frac{\left(f_{31}\right)^{2}+1}{2 f_{31}} \text { and } u_{331}=\frac{1}{m} \arccos h \frac{\left(f_{32}\right)^{2}+1}{-2 f_{32}} \text {. }
$$


This study shows that the $\left(\mathrm{G}^{\prime} / \mathrm{G}\right)$-expansion method is quite efficient and practically well suited in finding exact solutions for many equations. We hope that they will be useful for further studies in applied sciences.

\section{References}

[1] S.K. Liu, Z.T. Fu, S.D. Liu, Exact solutions to sine-Gordon-type equations, Phys. Lett.A 351 (2006) 59-63.

[2] H. New exact solutions for the sinh-Gordon equation. Chaos, Solitons Fractals 2006;28:489-96.

[3] A. Polyanin, V.F. Zaitsev, Handbook of Nonlinear Partial Differential Equations, FL:CRC, Boca Raton, 2004.2.

[4] A. Bekir, Application of the $\left(\mathrm{G}^{\prime} / \mathrm{G}\right)$-expansion method for nonlinear evolution equations, Phys. Lett. A 372 (19) (2008) 3400-3406.

[5] Liu H, Li J. Symmetry reductions, dynamical behavior and exact explicit solutions to the Gordon types of equations[J]. Journal of Computational and Applied Mathematics, 2014, 257: 144-156.

[6] M.L. Wang, X.Z. Li, J.L. Zhang, The (G'/G)-expansion method and travelling wave solutions of nonlinear evolution equations in mathematical physics, Phys. Lett. A 372(4) (2008) 417-423.

[7] L. Ling-Xiao, W. Ming-Liang, The $\left(\mathrm{G}^{\prime} / \mathrm{G}\right)$-expansion method and travelling wave solutions for a higher-order nonlinear schrdinger equation, Appl.Math. Comput. 208 (2009)440-445.

[8] S. Zhang, L. Dong, J. Ba, Y. Sun, The $\left(\mathrm{G}^{\prime} / \mathrm{G}\right)$-expansion method for nonlineardifferential-difference equations, Phys. Lett. A 373 (2009) 905-910. 